\title{
Ideal sedation for stroke thrombectomy: a prospective pilot single-center observational study
}

\author{
M. Asif Taqi, MD,, ${ }^{1,2}$ Sajid S. Suriya, MD, ${ }^{1}$ Ajeet Sodhi, MD,, ${ }^{1,2}$ Syed A. Quadri, MD, ${ }^{1}$ \\ Mudassir Farooqui, MD, ${ }^{3}$ Atif Zafar, MD, ${ }^{4}$ and Martin M. Mortazavi, MD1,2 \\ ${ }^{1}$ National Skull Base Foundation, Thousand Oaks; '2Los Robles Hospital and Medical Center, Thousand Oaks, California; \\ ${ }^{3}$ University of Oklahoma Health Sciences Center, Oklahoma City, Oklahoma; and ${ }^{4}$ University of New Mexico Health Sciences \\ Center, Albuquerque, New Mexico
}

OBJECTIVE Several retrospective studies have supported the use of conscious sedation (CS) over general anesthesia (GA) as the preferred methods of sedation for stroke thrombectomy, but a recent randomized controlled trial showed no difference in outcomes after CS or GA. The purpose of the Ideal Sedation for Stroke Thrombectomy (ISST) study was to evaluate the difference in time and outcomes in the reperfusion of anterior circulation in ischemic stroke using GA and monitored anesthesia care (MAC).

METHODS The ISST study was a prospective, open-label registry. A total of 40 patients who underwent mechanical thrombectomy for anterior circulation ischemic stroke were enrolled. Informed consent was obtained from each patient before enrollment. The primary endpoint included the interval between the patient's arrival to the interventional radiology room and reperfusion time. Secondary endpoints were evaluated to estimate the effects on the outcome of patients between the 2 sedation methods.

RESULTS Of the 40 patients, 32 received thrombectomy under MAC and 8 patients under GA. The male-to-female ratio was 18:14 in the MAC group and 4:4 in the GA group. The mean time from interventional radiology room arrival to reperfusion in the GA group was 2 times higher than that in the MAC group. Complete reperfusion ( $\mathrm{TICl}$ grade 3 ) was achieved in more than $50 \%$ of patients in both groups. The mean modified Rankin Scale score at 3 months was $<2$ in the MAC group and $>3$ in the GA group $(p=0.021)$.

CONCLUSIONS The findings from the pilot study showed a significantly shorter time interval between IR arrival and reperfusion and better outcomes in patients undergoing reperfusion for ischemic stroke in the anterior circulation using MAC compared with GA.

Clinical trial registration no.: NCT03036631 (clinicaltrials.gov)

https://thejns.org/doi/abs/10.3171/2018.11.FOCUS18522

KEYWORDS acute ischemic stroke; endovascular treatment; general anesthesia; monitored anesthesia care; conscious sedation

$\Lambda$ CUTE ischemic stroke (AIS) is caused by blockage of an intracranial blood vessel and accounts for $>$ $85 \%$ of all strokes. ${ }^{19}$ Medical treatment with anticoagulation therapy and antiplatelet therapy used to be standard protocol for thrombolysis in patients presenting with AIS caused by a large-vessel occlusion. The evolution of intraarterial treatment (IAT) with mechanical thrombectomy changed the paradigm of AIS management. Sev- eral randomized controlled trials (RCTs) have shown that the intraarterial treatment is superior to standard medical treatment alone. ${ }^{5,7,9,14,21}$ The clinical outcome after mechanical thrombectomy is contingent on timely recanalization of the intracranial blood vessel. ${ }^{20,24,25}$

The choice of sedation method in IAT has significant consequences on the timing of reperfusion and clinical outcome of thrombectomy. The options for sedation in-

ABBREVIATIONS AIS = acute ischemic stroke; $C S$ = conscious sedation; $D S A$ = digital subtraction angiography; GA = general anesthesia; $I A T$ = intraarterial treatment; IRR = interventional radiology room; ISST = Ideal Sedation for Stroke Thrombectomy; LAR = legally authorized representative; MAC = monitored anesthesia care; mRS = modified Rankin Scale; NIHSS = National Institutes of Health Stroke Scale; RCT = randomized controlled trial; $\mathrm{TICl}=\mathrm{Thrombolysis}$ in Cerebral Infarction.

SUBMITTED September 30, 2018. ACCEPTED November 14, 2018.

INCLUDE WHEN CITING DOI: 10.3171/2018.11.FOCUS18522. 
clude general anesthesia (GA) with intubation, conscious sedation (CS) without intubation, and monitored anesthesia care (MAC) without intubation. There is a paucity of evidence in the literature regarding the impact of choice of sedation on neurological outcome. In order to answer this key question, several retrospective studies were conducted and showed better outcomes with CS than with GA..$^{1-3,8,10,12,13,15}$

Three recently conducted RCTs in Europe, SIESTA (Sedation vs Intubation for Endovascular Stroke Treatment), AnStroke (Anesthesia during Stroke), and GOLIATH (General or Local Anesthesia in Intra Arterial Therapy), were more focused on neurological and functional outcomes and did not evaluate the effects of either type of sedation on the delay in reperfusion. ${ }^{16,22,23}$ Considering the lack of evidence on this critical point in acute stroke management, the Ideal Sedation for Stroke Thrombectomy (ISST) trial was designed to evaluate if GA with intubation delays the time from arrival into the interventional radiology room (IRR) to final reperfusion. ${ }^{28}$ To the best of our knowledge, this is the first prospective study conducted in the United States to compare the GA and MAC methods of sedation on the delay in the recanalization of anterior circulation ischemic stroke undergoing IAT.

\section{Methods}

\section{Study Design and Management}

The ISST trial was a single-center, open-label, prospective observational study conducted between October 2016 and April 2018. The study was approved by the Western IRB (Puyallup, WA) and was registered on clinicaltrials. gov (registration no. NCT03036631). All AIS patients undergoing IAT were screened for the study. Considering that this was a pilot study, a sample size of 40 patients was chosen and was approved by the IRB. Inclusion criteria were 1) age $\geq 18$ years; 2) suitable for IAT for large-vessel occlusion up to 8 hours from onset of stroke/last known well time; and 3) clinical equipoise for best mode of sedation. Exclusion criteria were 1) any known allergy to the sedation medication or its alternative; 2) any patient who had been intubated prior to screening; 3) posterior circulation stroke; and 4) pregnancy. Patients who met the criteria were offered to participate in the study. Informed consent was obtained before enrollment from either the patient or a legally authorized representative (LAR). Written informed consent was mandatory before any data collection. Baseline and procedural data points were collected from the patient's paper and electronic medical charts in a deidentified manner. Follow-up visits were done at 24 hours, at discharge/on day 7, day 30, and day 90 . The modified Rankin Scale (mRS) score was collected on day 30 and day 90 over telephone by certified study personnel (Fig. 1).

\section{Neurointervention Procedure}

All IAT procedures were performed at Los Robles Hospital, Thousand Oaks, California, by 2 neurointerventionalists, and anesthesia was initiated and monitored by anesthesiologists in all patients. Both neurointerventionalists are trained vascular neurologists with neurointervention training. The decision of choosing the anesthesia type was at the discretion of the neurointerventionalist and anesthesiologist. MAC was performed using fentanyl, midazolam, propofol, dexmedetomidine, or ketamine. The choice and combination of drugs were at the discretion of the anesthesiologist. GA was induced by administration of fentanyl or propofol and was maintained using sevoflurane or desflurane. Endotracheal intubation and induction of GA and its maintenance was managed by an anesthesiolo-

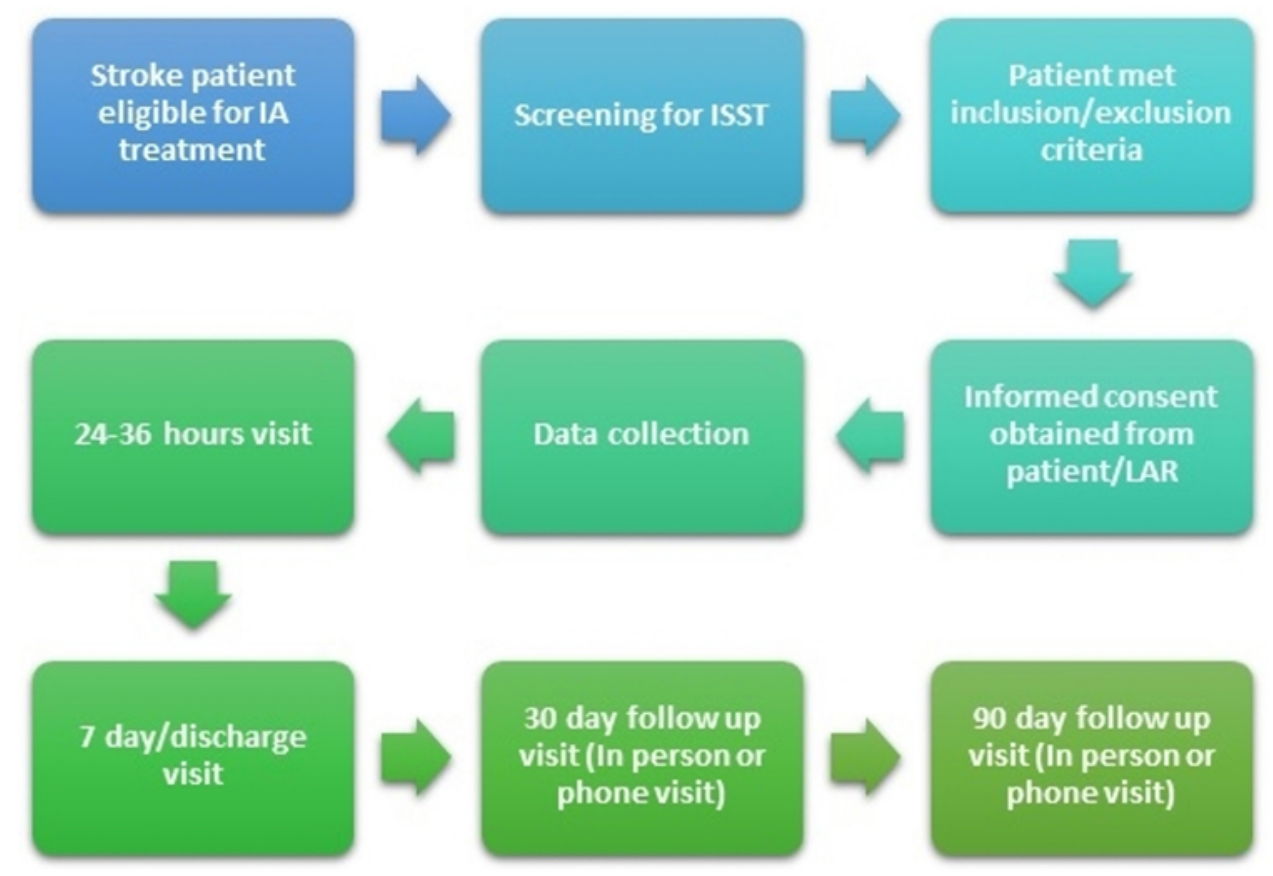

FIG. 1. Flowchart explaining the design of study. 
gist. The approach of thrombectomy was at the discretion of the neurointerventionalist based on the occlusion site, vascular tortuosity, and clot burden and included the use of a stent retriever, aspiration, or a combination of both. In patients with carotid artery stenosis or occlusion, carotid artery stenting and angioplasty was performed based on the discretion of the neurointerventionalist. The crucial time points (e.g., groin puncture time, reperfusion time) were recorded in a computerized log by interventional radiology staff. Reperfusion was assessed according to the Thrombolysis in Cerebral Infarction (TICI) grading system (grade 0, no perfusion; grade 1, perfusion past the initial obstruction, but limited distal branch filling with little or slow distal perfusion; grade 2a, perfusion of less than half of the vascular distribution of the occluded artery; grade $2 b$, perfusion of half or greater of the vascular distribution of the occluded artery; and grade 3 , full perfusion with filling of all distal branches). ${ }^{30}$

\section{Study Follow-Up and Outcomes}

The primary endpoint of the study was to evaluate if GA results in a delay in time to recanalize the occluded blood vessel measured by the time from arrival into the interventional radiology room to final reperfusion. The secondary endpoints were evaluated to compare the outcomes between MAC and GA and included reperfusion grade (TICI grade), National Institutes of Health Stroke Scale (NIHSS) score on days 1 and 7, mRS score on days 30 and 90, number of ICU days, number of passes performed, postprocedural asymptomatic or symptomatic subarachnoid hemorrhage or intracranial hemorrhage, and incidence of pneumonia.

Follow-up visits were performed at 24-36 hours and on day 7 or discharge (whichever comes first). On days 30 and 90 , the follow-up visits were conducted in person if the patient presented to the clinic; otherwise, follow-up was done over telephone by a study coordinator. Any adverse event was noted and reported during the visit.

\section{Statistical Analysis}

Data were entered in Excel (Microsoft Corp.) and analyzed using IBM SPSS (version 22, IBM Corp.). The final TICI grade after thrombectomy was expressed as frequency with percentages. Fisher's exact test was performed to find an association between the final TICI grade after thrombectomy and MAC and GA. The mean and standard deviation are reported for continuous variables, such as NIHSS score. While prior to running inferential analysis for continuous variables, normality was assessed by Shapiro-Wilk's test, which indicated that only 2 variables, IRR arrival time and final reperfusion time, were normally distributed. Therefore, comparison of these 2 variables between MAC and GA was checked using the independent samples t-test. The other continuous variables were compared using the Mann-Whitney U-test; $p<0.05$ was set as the level of significance.

\section{Results}

\section{Patient Characteristics}

A total of 79 patients who underwent mechanical
TABLE 1. Baseline demographics of patients stratified by anesthesia group

\begin{tabular}{lcc}
\hline & MAC Group & GA Group \\
\hline No. of patients & 32 & 8 \\
\hline Male/female ratio & $18: 14$ & $4: 4$ \\
\hline Mean age, yrs & 70 & 76 \\
\hline Transfer/ED admission ratio & $19: 13$ & $6: 2$ \\
\hline History of atrial fibrillation & 10 & 1 \\
\hline History of previous ischemic stroke & 4 & 0 \\
\hline Antiplatelet or anticoagulant at baseline & 12 & 2 \\
\hline
\end{tabular}

$\mathrm{ED}=$ emergency department.

Values are presented as the number of patients unless stated otherwise.

thrombectomy were screened for eligibility over the course of the registry. All patients met the inclusion criteria and did not fulfill the exclusion criteria. For 39 patients, informed consent could not be obtained due to: 1) patient was not competent to provide the consent; 2) LAR or family member was not available to provide the consent; or 3) the patient, LAR, or family member refused participation in the registry. Therefore, the final sample of 40 patients was included in the data analysis. Of these patients, 32 patients underwent mechanical thrombectomy under MAC and 8 patients underwent the procedure under GA, with male-to-female ratios of $18: 14$ and $4: 4$, respectively. The mean age was 70 years in the MAC group and 76 years in the GA group. The comparison of demographics and relevant previous medical history is outlined in Table 1.

All patients who were admitted through the emergency department underwent noncontrast CT scanning of the head on arrival. For patients with negative findings of hemorrhage or large-vessel occlusion, CTA of the head was performed. The site of the proximal level of occlusion based on initial noninvasive imaging findings is outlined in Fig. 2. The mean baseline NIHSS scores were 17.69 in the MAC group and 19.63 in the GA group, and the difference was not statistically significant $(p=0.539)$.

\section{Study Endpoints}

The mean time from IRR arrival to reperfusion for the

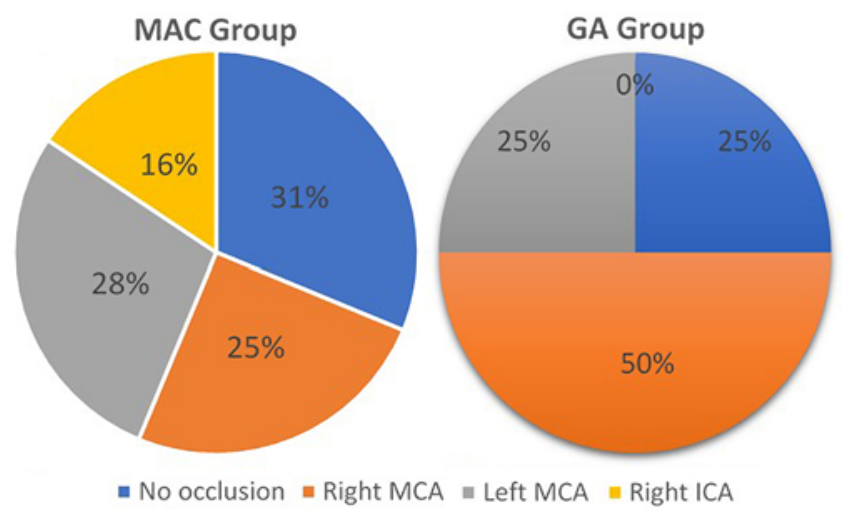

FIG. 2. Site of occlusion based on initial noninvasive imaging. ICA = internal carotid artery; MCA = middle cerebral artery. 
TABLE 2. Comparison of primary and secondary endpoints between the groups

\begin{tabular}{|c|c|c|c|c|c|}
\hline & \multicolumn{2}{|c|}{ MAC Group } & \multicolumn{2}{|c|}{ GA Group } & \multirow[b]{2}{*}{$\mathrm{p}$ Value } \\
\hline & Mean & SD & Mean & SD & \\
\hline Baseline NIHSS score & 17.69 & 5.99 & 19.63 & 5.83 & 0.539 \\
\hline IRR arrival to groin puncture time, mins & 14.81 & 5.01 & 21.62 & 5.95 & $0.002^{*}$ \\
\hline IRR to reperfusion time, mins & 62.03 & 26.84 & 101.37 & 41.38 & $0.002^{*}$ \\
\hline Total no. of passes & 1.97 & 1.62 & 2.88 & 1.96 & 0.117 \\
\hline NIHSS score at $24-36$ hrs & 5.65 & 6.85 & 9.75 & 6.48 & 0.092 \\
\hline NIHSS score on discharge/day 7 visit & 3.97 & 5.69 & 5.71 & 4.50 & 0.165 \\
\hline mRS score on discharge/day 7 visit & 2.26 & 2.07 & 3.63 & 1.60 & 0.162 \\
\hline No. of ICU days & 3.84 & 2.16 & 6.00 & 5.21 & 0.278 \\
\hline Length of stay in treating hospital & 9.69 & 14.06 & 18.25 & 15.15 & 0.025 \\
\hline mRS score on day 30 visit & 2.05 & 1.86 & 4.00 & 0.82 & 0.02 \\
\hline mRS score on day 90 visit & 1.64 & 1.68 & 3.29 & 0.95 & 0.021 \\
\hline Postthrombectomy final $\mathrm{TICl}$ score & \multicolumn{4}{|c|}{ No. of Patients (\%) } & $0.702 \dagger$ \\
\hline $2 b$ & \multicolumn{2}{|c|}{$13(40.6 \%)$} & \multicolumn{2}{|c|}{$4(50 \%)$} & \\
\hline 3 & \multicolumn{2}{|c|}{$19(59.4 \%)$} & \multicolumn{2}{|c|}{$4(50 \%)$} & \\
\hline
\end{tabular}

GA group was about 2 times higher than for the MAC group $(p=0.002)$. Similarly, the mean time from IRR arrival to groin puncture was significantly shorter $(\mathrm{p}=$ 0.002 ) in the MAC group than in the GA group (15 and 21 minutes, respectively; Table 2 and Fig. 3).

A final TICI grade of $2 \mathrm{~b}$ or more after thrombectomy was achieved in the entire cohort. A TICI grade of 3 was achieved in $60 \%$ of the MAC group and $50 \%$ of the GA group (Table 2). However, the mean final TICI grade was not different between the groups.

The mean NIHSS score at the 24- to 36-hour visit for the MAC group was slightly less than that for the GA group (4 and 5.7, respectively). Similarly, the mRS score on discharge was also lower in the MAC group $(<3)$ than in the GA group ( $>3$ ); however, this difference was not statistically significant. The mean mRS scores at the 30and 90-day visits were significantly different between the MAC and GA groups ( $\mathrm{p}=0.02$ and 0.021 , respectively). The number of ICU days was similar between the groups, whereas the mean length of hospital stay for the MAC group was significantly shorter (9.7 days) than for the GA group (15.2 days; $p=0.025$ ).

Of the 32 patients in the MAC group, 2 (3\%) patients developed pneumonia in the 7-day postthrombectomy period, whereas in the GA group, only $1(12 \%)$ patient developed pneumonia, who died of respiratory failure. One patient in the MAC group developed symptomatic right parenchymal hematoma within 48 hours after the procedure.

\section{Discussion}

IAT is a method that involves catheterization of intracranial blood vessels with the guidance of digital subtraction angiography (DSA), which subtracts bone from the images, enabling visualization of the blood vessels. Using intracranial catheters, clots can be dissolved with intraarterial administration of thrombolytics or can be removed mechanically using a stent retriever. The optimal subtraction on DSA requires a stable head position; any unusual movements of the head can affect visibility of the blood vessels and clot. ${ }^{4}$ Since IAT has been established as standard-of-care for large-vessel occlusion, significant efforts are underway to streamline the procedure to achieve recanalization with the shortest possible time from IRR arrival to groin puncture..$^{27,29}$ One unresolved aspect of the IAT procedure is to have minimal effects of anesthesia on the timely reperfusion of blood vessels.

Overall, GA is the preferred choice of anesthesia due to the fact that it is associated with less patient anxiety and agitation and a lower risk of aspiration pneumonia. ${ }^{17}$ It also provides an additional advantage of better visualization of the clot and retrieval on first pass due to the stable position of the patient's head, which can reduce the groin puncture to reperfusion time. ${ }^{17}$ On the other hand, GA causes a decrease in blood pressure during induction, hypocapnia causing vasoconstriction, and delay in time to groin puncture. ${ }^{26}$ In a study by Herrmann et al., the authors found a delay in initiation of IAT for acute stroke even with a fasttrack intubation protocol. ${ }^{11}$ To answer this key question, a few retrospective studies were done that have demonstrated better outcomes with CS than with GA. ${ }^{1-3,8,10,12,13,15}$ Given the clinical equipoise, the first RCT, the SIESTA trial, was designed by Schönenberger et al., followed by the AnStroke trial, and more recently the GOLIATH trial was conducted in Europe. ${ }^{16,22,23}$

In the SIESTA trial, the authors found no significant improvement in neurological status at 24 hours, as measured by the NIHSS. In the AnStroke trial, which was published in 2017, the authors found no significant difference in neurological outcome 3 months after thrombectomy between the 2 groups. ${ }^{16}$ This trial also showed no statistically significant difference in the time from CT to 


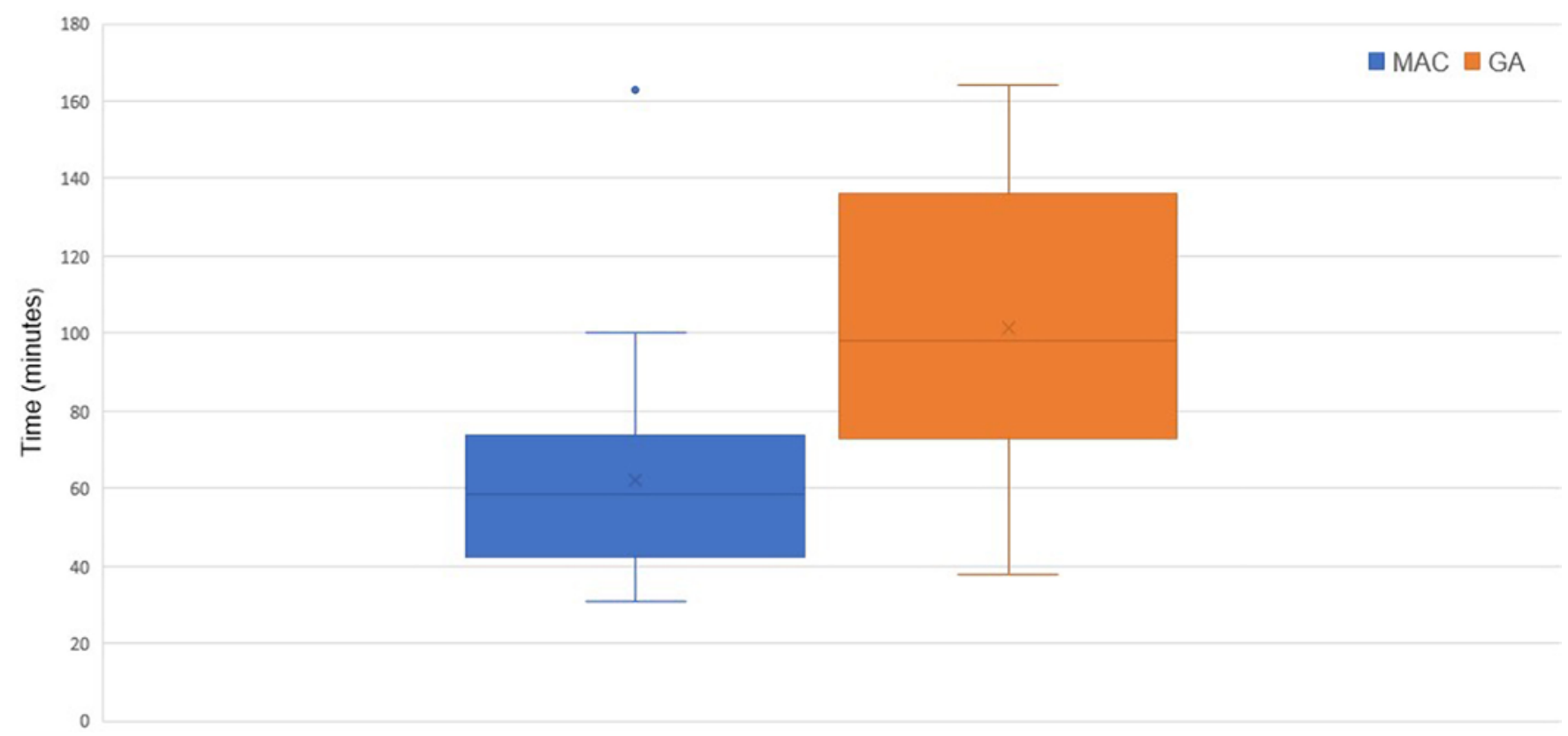

FIG. 3. Comparison of IRR arrival to reperfusion time in the MAC and GA groups. The box represents the IQR. The line and $x$ inside the box represent the median and mean, respectively. The whiskers represent the minimum-maximum values.

groin puncture between the GA and CS groups. These 2 trials showed no difference in functional and neurological outcomes of patients between the groups. Another RCT, the GOLIATH trial, was published recently by Simonsen et al. ${ }^{23}$ In this study, the authors examined the effects of mode of sedation on the infarct growth after mechanical thrombectomy as a primary endpoint and found no statistically significant difference between the 2 groups. ${ }^{23}$ All of these RCTs were focused on neurological and functional outcomes, and they did not address the impact of the modes of sedation on timing and the delay in reperfusion.

ISST was a single-center, prospective pilot study that focused on the comparison of timings between the MAC and GA groups. The results showed a decreased time from IRR arrival to reperfusion in the MAC group compared with the GA group. Although post hoc analysis of the SIESTA trial showed a shorter time from groin puncture to reperfusion in the GA group, the authors reported a 10 -minute delay in the time from door to groin puncture in this group. ${ }^{18}$ Similarly, the MR CLEAN (Multicenter Randomized Clinical Trial of Endovascular Treatment for Acute Ischemic Stroke in The Netherlands) trial by Berkhemer et al. reported poor outcomes and a delay of 32 minutes with GA versus non-GA. ${ }^{6}$ In our study, the mean IRR arrival to groin puncture time was significantly shorter in the MAC group due to the intubation-related delays in the GA group. On the other hand, GA is associated with a shorter time interval between the groin puncture time and reperfusion time due to better visualization of the clot since the patient's head is stable. ${ }^{4}$ Therefore, to evaluate the overall effects of GA and MAC, in the ISST study, the interval between IRR arrival and final reperfusion time was compared and showed that earlier reperfusion occurred after MAC.

Furthermore, our study showed significantly better out- come, as indicated by the mRS score at 3 months in the MAC group than in the GA group $(\mathrm{p}=0.021)$. Previous retrospective studies showed better outcome in the CS group, whereas recent RCTs have shown no statistically significant difference in outcomes between GA and CS. ${ }^{1-3,8,10,12}$, ${ }_{13,15}$ Patients in the MAC group had lower NIHSS scores at discharge than patients in the GA group; however, this difference was not statistically significant. The SIESTA trial showed no significant difference in NIHSS between the 2 groups at the 24-hour follow-up.22 Also, patients in the GA group spent more days in the ICU due to to intubation. The SIESTA trial showed a higher postprocedural frequency of pneumonia, and the results were in line with those of previous retrospective studies. ${ }^{1-3,8,10,12,13,15}$ In our study, 2 patients developed postprocedural pneumonia in the MAC group while 1 patient in the GA group developed aspiration pneumonia. In the MAC group, 4 patients died at discharge/day-7 visit (cardiopulmonary arrest [ $\mathrm{n}=$ $2]$, pancreatic cancer [n=1], and sepsis $[n=1])$. In the GA group, 1 patient died of respiratory failure beyond the day-30 visit due to a comorbid condition. These deaths were unrelated to the method of anesthesia or the thrombectomy procedure and thus were included in the 90-day outcome analysis.

In our study, the patients in the MAC group received fewer passes than those in the GA group, but the difference was not statistically significant $(p=0.117)$. The higher number of passes in thrombectomy relates to a delay in reperfusion and could result in a worse outcome, as evident in the TREVO (Thrombectomy Revascularization of Large Vessel Occlusions in Acute Ischemic Stroke) study. ${ }^{31}$ After thrombectomy, a TICI grade of 3 was achieved in more than half of the patients in both groups, which infers no impact of either mode of sedation on clot retrieval. The results of the TICI grade after thrombectomy in our 
study are in contrast to those in the GOLIATH trial, which showed a higher rate of TICI grade $\geq 2 \mathrm{~b}$ among patients in the GA group. As shown in retrospective studies and RCTs, our study also showed a higher incidence of pneumonia and longer hospital stay in the GA group.

\section{Study Merits and Limitations}

ISST is the first prospective study conducted in North America to report the effects of sedation on the reperfusion time and outcomes. Previous studies, including European RCTs, compared the outcomes between CS and GA groups. Our pilot study focused on the comparison between MAC and GA, and, to the best of our knowledge, it is the only study comparing these 2 groups and focusing on the IRR arrival to reperfusion time between these two modes of sedation.

Since our study was a pilot project, the sample size was kept small and it was conducted at a single center. However, the major limitation of our study is that it was a prospective registry instead of an RCT. The randomization of patients was limited since most of the stroke patients were not competent enough to provide consent before the procedure. Also, in most cases, the family or LAR was not available to provide the consent. In order to conduct an RCT, a waiver of consent will be required to randomize patients before the procedure. These points should be kept in mind while designing a future RCT in the United States.

\section{Conclusions}

The ISST study showed not only less delay in time to reperfusion with MAC but also showed better neurological outcomes compared with GA. A larger RCT comparing all 3 modes of sedation (MAC, GA, and CS) and their effects on outcome and early reperfusion is required. The results from our pilot study will help to design a large, multicenter RCT.

\section{References}

1. Abou-Chebl A, Lin R, Hussain MS, Jovin TG, Levy EI, Liebeskind DS, et al: Conscious sedation versus general anesthesia during endovascular therapy for acute anterior circulation stroke: preliminary results from a retrospective, multicenter study. Stroke 41:1175-1179, 2010

2. Abou-Chebl A, Yeatts SD, Yan B, Cockroft K, Goyal M, Jovin T, et al: Impact of general anesthesia on safety and outcomes in the endovascular arm of Interventional Management of Stroke (IMS) III trial. Stroke 46:2142-2148, 2015

3. Abou-Chebl A, Zaidat OO, Castonguay AC, Gupta R, Sun CHJ, Martin CO, et al: North American SOLITAIRE StentRetriever Acute Stroke Registry: choice of anesthesia and outcomes. Stroke 45:1396-1401, 2014

4. Ahn SH, Prince EA, Dubel GJ: Basic neuroangiography: review of technique and perioperative patient care. Semin Intervent Radiol 30:225-233, 2013

5. Berkhemer OA, Fransen PS, Beumer D, van den Berg LA, Lingsma HF, Yoo AJ, et al: A randomized trial of intraarterial treatment for acute ischemic stroke. N Engl J Med 372:11-20, 2015

6. Berkhemer OA, van den Berg LA, Fransen PS, Beumer D, Yoo AJ, Lingsma HF, et al: The effect of anesthetic management during intra-arterial therapy for acute stroke in MR CLEAN. Neurology 87:656-664, 2016

7. Campbell BC, Mitchell PJ, Kleinig TJ, Dewey HM, Churilov
L, Yassi N, et al: Endovascular therapy for ischemic stroke with perfusion-imaging selection. N Engl J Med 372:10091018,2015

8. Davis MJ, Menon BK, Baghirzada LB, Campos-Herrera CR, Goyal M, Hill MD, et al: Anesthetic management and outcome in patients during endovascular therapy for acute stroke. Anesthesiology 116:396-405, 2012

9. Goyal M, Demchuk AM, Menon BK, Eesa M, Rempel JL, Thornton J, et al: Randomized assessment of rapid endovascular treatment of ischemic stroke. N Engl J Med 372:10191030,2015

10. Hassan AE, Chaudhry SA, Zacharatos H, Khatri R, Akbar $\mathrm{U}$, Suri MFK, et al: Increased rate of aspiration pneumonia and poor discharge outcome among acute ischemic stroke patients following intubation for endovascular treatment. Neurocrit Care 16:246-250, 2012

11. Herrmann O, Hug A, Bösel J, Petersen JJ, Hartmann M, Rohde S, et al: Fast-track intubation for accelerated interventional stroke treatment. Neurocrit Care 17:354-360, 2012

12. Jagani M, Brinjikji W, Rabinstein AA, Pasternak JJ, Kallmes DF: Hemodynamics during anesthesia for intra-arterial therapy of acute ischemic stroke. J Neurointerv Surg 8:883-888, 2016

13. John S, Thebo U, Gomes J, Saqqur M, Farag E, Xu J, et al: Intra-arterial therapy for acute ischemic stroke under general anesthesia versus monitored anesthesia care. Cerebrovasc Dis 38:262-267, 2014

14. Jovin TG, Chamorro A, Cobo E, de Miquel MA, Molina CA, Rovira A, et al: Thrombectomy within 8 hours after symptom onset in ischemic stroke. N Engl J Med 372:2296-2306, 2015

15. Jumaa MA, Zhang F, Ruiz-Ares G, Gelzinis T, Malik AM, Aleu A, et al: Comparison of safety and clinical and radiographic outcomes in endovascular acute stroke therapy for proximal middle cerebral artery occlusion with intubation and general anesthesia versus the nonintubated state. Stroke 41:1180-1184, 2010

16. Löwhagen Hendén P, Rentzos A, Karlsson JE, Rosengren L, Leiram B, Sundeman H, et al: General anesthesia versus conscious sedation for endovascular treatment of acute ischemic stroke: the AnStroke trial (anesthesia during stroke). Stroke 48:1601-1607, 2017

17. McDonagh DL, Olson DM, Kalia JS, Gupta R, Abou-Chebl A, Zaidat OO: Anesthesia and sedation practices among neurointerventionalists during acute ischemic stroke endovascular therapy. Front Neurol 1:118, 2010

18. Pfaff JAR, Schönenberger S, Nagel S, Ringleb PA, Hacke W, Bendszus M, et al: Effect of general anesthesia versus conscious sedation for stroke thrombectomy on angiographic workflow in a randomized trial: a post hoc analysis of the SIESTA trial. Radiology 286:1016-1021, 2018

19. Powers WJ, Derdeyn CP, Biller J, Coffey CS, Hoh BL, Jauch EC, et al: 2015 American Heart Association/American Stroke Association focused update of the 2013 guidelines for the early management of patients with acute ischemic stroke regarding endovascular treatment. Stroke 46:3020-3035, 2015

20. Rha JH, Saver JL: The impact of recanalization on ischemic stroke outcome: a meta-analysis. Stroke 38:967-973, 2007

21. Saver JL, Goyal M, Bonafe A, Diener HC, Levy EI, Pereira VM, et al: Stent-retriever thrombectomy after intravenous t-PA vs. t-PA alone in stroke. N Engl J Med 372:2285-2295, 2015

22. Schönenberger S, Uhlmann L, Hacke W, Schieber S, Mundiyanapurath S, Purrucker JC, et al: Effect of conscious sedation vs general anesthesia on early neurological improvement among patients with ischemic stroke undergoing endovascular thrombectomy: a randomized clinical trial. JAMA 316:1986-1996, 2016

23. Simonsen CZ, Yoo AJ, Sørensen LH, Juul N, Johnsen SP, Andersen G, et al: Effect of general anesthesia and conscious 
sedation during endovascular therapy on infarct growth and clinical outcomes in acute ischemic stroke: a randomized clinical trial. JAMA Neurol 75:470-477, 2018

24. Smith WS: Intra-arterial thrombolytic therapy for acute basilar occlusion: pro. Stroke 38 (2 Suppl):701-703, 2007

25. Smith WS: Safety of mechanical thrombectomy and intravenous tissue plasminogen activator in acute ischemic stroke. Results of the multi Mechanical Embolus Removal in Cerebral Ischemia (MERCI) trial, part I. AJNR Am J Neuroradiol 27:1177-1182, 2006

26. Takahashi C, Liang CW, Liebeskind DS, Hinman JD: To tube or not to tube? The role of intubation during stroke thrombectomy. Front Neurol 5:170, 2014

27. Taqi M, Quadri S, Suriya S: E-058 pilot study: Feasibility of the use of Ventura emergency large vessel occlusion scale to identify large vessel occlusion stroke. J Neurointerv Surg 8:A74, 2016 (Abstract)

28. Taqi M, Sodhi A, Suriya S, Adl F, Farooqi M, Quadri S, et al: E-134 Ideal sedation for stroke thrombectomy: early findings of a prospective registry. J Neurointerv Surg 10:A114A115, 2018 (Abstract)

29. Taqi M, Sodhi A, Suriya S, Quadri S, Shepherd D, Salvucci A, et al: O-028 Reliability of Ventura emergency large vessel occlusion scale; a retrospective review. J Neurointerv Surg 9:A15-A16, 2017 (Abstract)

30. Tomsick T, Broderick J, Carrozella J, Khatri P, Hill M, Palesch Y, et al: Revascularization results in the Interventional Management of Stroke II trial. AJNR Am J Neuroradiol 29:582-587, 2008

31. Zaidat OO, Castonguay AC, Nogueira RG, Haussen DC, English JD, Satti SR, et al: TREVO stent-retriever mechanical thrombectomy for acute ischemic stroke secondary to large vessel occlusion registry. J Neurointerv Surg 10:516-524, 2018

\section{Disclosures}

A financial research grant was provided by Stryker Neurovascular to support this study.

Dr. Taqi: consultant for Stryker Neurovascular. Dr. Mortazavi: key opinion leader for Haag-Streit and consultant for DePuy Synthes, American Surgical Company, and Fiagon.

\section{Author Contributions}

Conception and design: Taqi. Acquisition of data: Taqi, Suriya, Sodhi, Quadri. Analysis and interpretation of data: Taqi, Suriya, Quadri, Farooqui, Zafar, Mortazavi. Drafting the article: all authors. Critically revising the article: all authors. Reviewed submitted version of manuscript: all authors. Approved the final version of the manuscript on behalf of all authors: Taqi. Statistical analysis: Suriya, Farooqui. Administrative/technical/material support: Suriya, Sodhi, Quadri. Study supervision: Taqi, Mortazavi.

\section{Supplemental Information}

\section{Previous Presentations}

Early findings of this study were presented as an e-poster at the Society of NeuroInterventional Surgery (SNIS) 15th Annual Meeting and Fellows Course, San Francisco, July 23-27, 2018.

\section{Correspondence}

M. Asif Taqi: National Skull Base Foundation, Thousand Oaks, CA. asiftaqi@icloud.com. 